\title{
Communication
}

\section{Molecularly Imprinted Microspheres for the Adsorption of Chlorpyrifos}

\author{
Wei Yao ${ }^{1}$, Shanyu Wang ${ }^{1}$, Baofeng Xu ${ }^{1}$, Hong Yin ${ }^{1}$, Zhixian Gao, * \\ ${ }^{1}$ Center for Disease Control and Prevention, Agency for Offices Administration, Beijing, China \\ ${ }^{2}$ Institute of Hygiene and Environmental Medicine, Tianjin, China
}

Email address:

playaowei@126.com (Zhixian Gao)

${ }^{*}$ Corresponding author

\section{To cite this article:}

Wei Yao, Shanyu Wang, Baofeng Xu, Hong Yin, Zhixian Gao. Molecularly Imprinted Microspheres for the Adsorption of Chlorpyrifos. International Journal of Materials Science and Applications. Vol. 5, No. 3, 2016, pp. 160-163. doi: 10.11648/j.ijmsa.20160503.17

Received: June 14, 2016; Accepted: June 28, 2016; Published: July 13, 2016

\begin{abstract}
To obtain the desired specific adsorbents for chlorpyrifos to enrichment, separation and analysis of trace pesticides residue, molecularly imprinted polymer microspheres were prepared by precipitation polymerization using chlorpyrifos and methacylic acid (MAA) as template and functional monomer respectively. Computer simulation was employed to demonstrate the mechanism of the interaction between chlorpyrifos and MAA. The adsorption properties of chlorpyrifos, in acetonitrile for imprinted microspheres were evaluated by equilibrium rebinding experiments. Scatchard plot analysis revealed that there was a class of binding sites populated in the molecularly imprinted polymers (MIPs). The specificity of the imprinted microspheres was investigated by binding analysis using chlorpyrifos and related organophosphate pesticides. The results indicated that the obtained imprinted microspheres showed a good selectivity for chlorpyrifos.
\end{abstract}

Keywords: Adsorption, Chlorpyrifos, Molecularly Imprinted Polymers, Precipitation Polymerization

\section{Introduction}

Chlorpyrifos, a kinds of organophosphate pesticides, is most frequently used to control insect and pests on agricultural and vegetable crops, due to the lower environmental persistence. However, they have high acute toxicity to human health and ecosystems [1]. Standard analytical methods, such as high performance liquid chromatography (HPLC), gas chromatography-mass spectrometry (GC-MS) have been commonly used for an efficient determination of chlorpyrifos and its metabolites [2, 3]. However, the trace pesticides residues in real samples must be cleaned up and enriched prior to the detection by GC or HPLC, and this process takes $50-75 \%$ of the total time of the analysis [4]. Thus, the development of adsorbents with good-selectivity and excellent stability to certain carbamate pesticides is necessary.

During the last few years, molecularly imprinted polymers (MIPs) have been used as synthetic materials that have considerable molecular recognition ability, and can be used as a separation media. MIPs have been utilized in solid phase extraction [5], sensor [6] and chromatography for their potential absorptive selectivity to the target molecules and the related compounds [7-9].

The present work describes the preparation of chlorpyrifos-imprinted polymer using chlorpyrifos as template, methacrylic acid (MAA) as functional monomer, ethylene glycol dimethacrylate (EGDMA) as cross-linker, azobisisobutyronitrile (AIBN) as initiator and acetonitrile as porogen, respectively. Computer modelling was employed to demonstrate the interaction between template and function monomer. The adsorption properties were evaluated by equilibrium rebinding experiments and Scatchard analysis. The selectivity of the obtained particles was elucidated by the different rebinding capability of the chlorpyrifos and the related compounds. Desired pesticide imprinted polymer microspheres were obtained for the separation, enrichment and analysis of trace pesticides residue. 


\section{Experimenal}

\subsection{Materials and Reagents}

Chlorpyrifos, dimethoate, omethoate and dichlorvos were purchased from ChemService (USA). Methacrylic acid (MAA) and ethylene glycol dimethacrylate (EGDMA) were supplied by Sigma. 2, 2-Azobisiobutyronitrile (AIBN) was supplied by Aldrich. All the other chemicals were obtained from Tianjin Chemical Co, Ltd (China) in analytical grade.

\subsection{Molecular Modelling of Monomer-Template Interactions}

The server used to simulate monomer-template interactions was a Silicon Graphics Origin 350 running IRIX 6.5 operating system. The server was configured with four $4700 \mathrm{MHz}$ reduced instruction set processors; 2GB memory and a 72GB fixed drive. This system was used to execute the software packages SYBYL 6.9 (Tripos, St. Louis, MO, USA). The computational design was performed in three steps [10]. In the first step, the molecular model of chlorpyrifos (template) and the molecular model of the most commonly used functional monomer MAA were designed. These structures were charged using the molecular mechanics method applying an energy minimization with the MINIMIZE command. In the second step, the Leapfrog algorithm was applied to investigate the possible interactions between the template and functional monomer. The program was activated for 600,000 steps. The results from the runs were examined evaluating the empirical binding scores.

\subsection{Preparation of Imprinted Polymers}

Chlorpyrifos $(0.5 \mathrm{mmol})$ and MAA $(2 \mathrm{mmol})$ were dissolved in $40 \mathrm{~mL}$ acetonitrile in a $50 \mathrm{ml}$ round-bottom flask, then oscillated for $12 \mathrm{~h}$ at room temperature. The EGDMA (10mmol) and the AIBN (10mg) were added. After purging with nitrogen for $5 \mathrm{~min}$ to remove oxygen, the flask was sealed and allowed to polymerize in a thermostated water bath at $60^{\circ} \mathrm{C}$ for $24 \mathrm{~h}$. The polymers were collected by centrifugation. The polymer particles were extensively washed with methanol/acetic acid $(9: 1)$ until the template molecule could not be detected in the extraction solvent. Then the particles were washed several times with methanol until the $\mathrm{pH}$ of the extracted solvent was neutral. Finally the solvent was removed by centrifugation and the particles were dried under vacuum for later use.

Non-imprinted polymers (Non-MIPs) were obtained following the same procedures with the absence of template molecule.

\subsection{Rebind Study of MIPs and Scatchard Analysis}

The saturation binding experiments and the subsequent Scatchared analysis were carried out to confirm rebind performance. The polymer particles $(20 \mathrm{mg})$ were mixed with a $2.0 \mathrm{ml}$ solution containing a known concentration of chlorpyrifos $(0.05-1.0 \mathrm{mmol} / \mathrm{L})$. The mixtures were incubated for $20 \mathrm{~h}$ under continuous shaking at room temperature and centrifuged using an eppendorf high-speed centrifuge. The supernatant was withdrawn and the concentration of chlorpyrifos was determined by GC. Data from triplicate measurements were averaged for the Scatchard analysis.

The selectivity of the MIPs was investigated using chlorpyrifos and the structurally related compounds' adsorption on the MIPs and Non-MIPs.

\section{Results and Discussion}

\subsection{Preliminary Microspheres Evaluation}

At present, the typical preparation method for MIPs is bulk polymerization, but the polymer prepared by this method is far from ideal as a random shape. There are many reasons for the breakage for the crushing and sieving in the particle preparation and the shrinking in the removal of the template molecule with polar organic solvents. The advent of the polymerization strategies, such as, suspension polymerization [11], precipitation polymerization [12, 13], can overcome these drawbacks. Among them, precipitation polymerization is the easiest method, which is just carried out in the presence of a higher amount of porogen (compared with the traditional methods) without stirrer and dispersant. As a dilute monomer solution has been used as a starting solution during precipitation polymerization, uniformly distributed microspheres (about 200nm in diameter) were obtained.

\subsection{Computer Modelling}

With the development of chemometrics and computer science, the computer modelling on the interactions between atoms is available. So in this work, the interaction between chlorpyrifos and MAA was calculated by this technology. The molecular model of chlorpyrifos and MAA were designed. As can be seen in Fig. 1, hydrogen bond defined the molecular complex between the oxygen atom of chlorpyrifos and the hydroxyl group of MAA, the distances between them were $2.458 \AA$ and $2.155 \AA$, respectively. Therefore, the creation of selectivity cavities for chlorpyrifos in the polymers deemed to be possible.

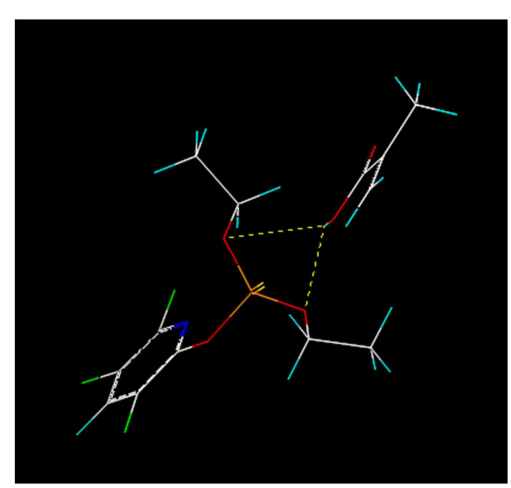

Figure 1. The computational design structures of chlorpyrifos-MAA complexes.

\subsection{Adsorption Analysis}

In order to investigate the affinity of the imprinted microspheres for chlorpyrifos, binding experiments and 
subsequent Scatchard analysis were carried out. The binding isotherms of chlorpyrifos to the MIPs and Non-MIPs determined in the range of $0.05 \mathrm{mmol} / \mathrm{L}$ to $1.0 \mathrm{mmol} / \mathrm{L}$ (Fig. 2). The amount of chlorpyrifos bound to MIPs at equilibrium experiment increased along with increasing the initial concentration of chlorpyrifos and the amount on MIPs is larger than that on Non-MIPs, which suggested that chlorpyrifos binding to imprinted microspheres might be caused by the specific binding to a certain number of binding sites populated in the imprinted microspheres.

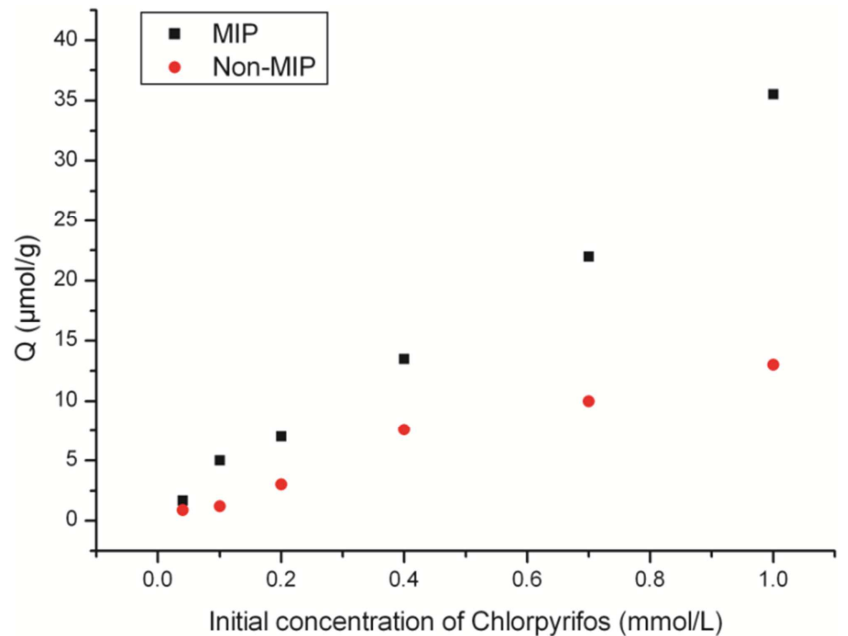

Figure 2. Isotherm of chlorpyrifos adsorption on the MIPs and Non-MIPs. $Q$ : the amount of bound chlorpyrifos on per gram dry microspheres; Amount of the polymers: $20 \mathrm{mg}$; incubation time: $20 \mathrm{~h} ; \mathrm{V}=2.0 \mathrm{~mL}$; at room temperature; $n=3$.

The saturation binding data were further processed with Scatchard equation to estimate the binding properties of chlorpyrifos on the MIPs. The Scatchard equation was as follows:

$$
Q / C=Q_{\max }-Q / K_{D}
$$

Where $Q$ is the amount of chlorpyrifos bound to the MIPs; $C$ is the equilibrium concentration of chlorpyrifos; $Q_{\max }$ is the apparent maximum number of binding sites and $K_{\mathrm{D}}$ is equilibrium dissociation constant. $Q_{\max }$ and $K_{\mathrm{D}}$ can be determined from the intercept and slope, respectively, when $Q / C$ is plotted verus $Q$.

Fig. 3 showed that the Scatchard plots were only a single straight line, which illustrated that there was only a class of binding sites populated in the MIPs. Based on the slope and intercept of each Scatchard plot line of the MIPs, the apparent maximum number $\left(Q_{\max }\right)$ and equilibrium dissociation constant $\left(K_{\mathrm{D}}\right)$ of the affinity-binding sites have been calculated. The results given in Table 1 showed that the apparent maximum number $\left(Q_{\max }\right)$ of MIPs is higher than that of Non-MIPs. It was supposed that the affinity towards chlorpyrifos during the rebinding experiment is due to a suitable matching stereo arrangement and the interaction of function monomers at MIPs binding sites.

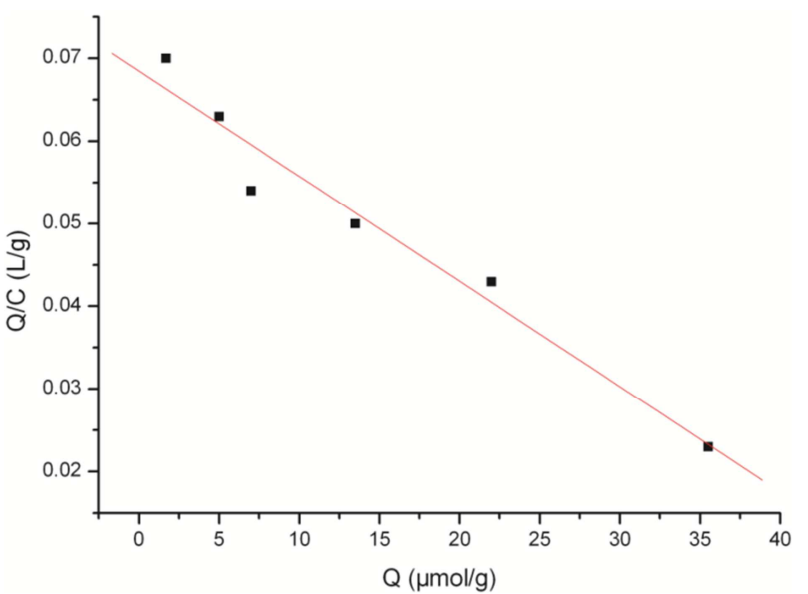

Figure 3. Scatchard plot of chlorpyrifos rebinding on the MIPs. Q: the amount of bound chlorpyrifos on per gram dry polymers; $C$ : concentration of free chlorpyrifos at adsorption equilibrium.

Table 1. The dissociation constants $\left(K_{D}\right)$ and maximum number $\left(Q_{\max }\right)$ of binding site for MIPs and Non-MIPs.

\begin{tabular}{lll}
\hline \multirow{2}{*}{ Polymers } & \multicolumn{2}{l}{ Binding sites } \\
\cline { 2 - 3 } & $\boldsymbol{K}_{\mathbf{D}}(\mathbf{m o l} / \mathbf{L})$ & $\boldsymbol{Q}_{\max }(\boldsymbol{\mu} \mathbf{m o l} / \mathbf{g})$ \\
\hline MIPs & $7.87 \times 10^{-3}$ & 53.91 \\
Non-MIPs & $3.75 \times 10^{-3}$ & 31.22 \\
\hline
\end{tabular}

\subsection{Binding Selectivity of the Imprinted Microspheres}<smiles>CCOP(=S)(OCC)Oc1nc(Cl)c(Cl)cc1Cl</smiles><smiles>CNC(=O)CSP(=S)(OC)OC</smiles>

Clilor pyrifos

Dincthoate<smiles>CNC(=O)CSP(=O)(OC)OC</smiles><smiles>COP(=O)(OC)OC=C(Cl)Cl</smiles>

(inethoate

Dichlorvos

Figure 4. Structures of the organophosphorous pesticides used in this study.

Table 2. Adsorption selectivity of the MIPs and Non-MIPs.

\begin{tabular}{lll}
\hline \multirow{2}{*}{ Analytes } & \multicolumn{2}{c}{$\boldsymbol{Q}(\boldsymbol{\mu} \mathbf{m o l} / \mathbf{g})$} \\
\cline { 2 - 3 } & MIPs & Non-MIPs \\
\hline Chlorpyrifos & $16.2 \pm 1.7$ & $7.6 \pm 2.2$ \\
Dimethoate & $7.3 \pm 2.1$ & $6.3 \pm 0.9$ \\
Omethoate & $7.2 \pm 0.6$ & $6.6 \pm 0.3$ \\
Dichlorvos & $8.1 \pm 1.2$ & $7.2 \pm 1.3$ \\
\hline
\end{tabular}

MIPs and Non-MIPs: 20mg, respectively; initial concentration of analyte: $0.5 \mathrm{mmol} / \mathrm{L}$; solvent: acetonitrile; volume: $2 \mathrm{~mL}$; incubation time: $20 \mathrm{~h}$, at room temperature; $\mathrm{n}=3$.

In order to verify whether the imprinted polymer microspheres are selective to chlorpyrifos, the binding experiments of chlorpyrifos and structurally related pesticide to the microspheres were conducted (The structures are listed in Fig. 4). As can be seen from the results in table 2 that the MIPs 
obviously exhibited high binding affinity for chlorpyrifos. As for the non-imprinted polymer microspheres, they showed considerably less binding for the analytes. From Fig. 4, there are some structural differences among the analytes. The selectivity of the imprinted polymer microspheres might also give some insights into the molecular recognition mechanism in molecularly imprinted polymer. The functional group and shape complementarity between the template molecules and the structure at the binding sites in the imprinted polymer contribute to the template adsorption onto the MIPs. From the results of computer design and adsorption experiments of the MIPs, the possible polymerization procedure of chlorpyrifos-MIP can be concluded as follows. (Fig. 5)

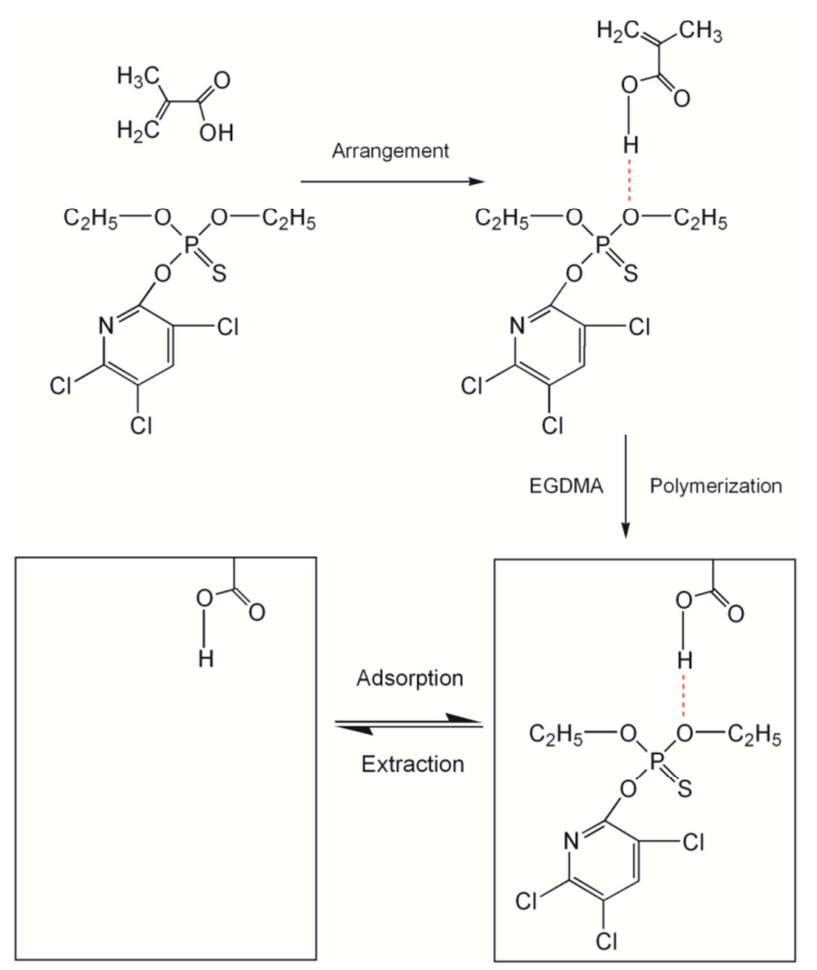

Figure 5. The possible polymerization procedure of chlorpyrifos-MIP in acetonitrile.

\section{Conclusions}

In the present work, desired uniform chlorpyrifos imprinted polymer microspheres were synthesized with satisfactory yield by precipitation polymerization. The interaction mechanism of monomer and chlorpyrifos was simulated by computer design. The studies will help us to further understand the recognition mechanism in molecular imprinted polymers and the template molecules. Equilibrium binding experiment was employed to investigate the adsorption properties of the obtained microspheres for chlorpyrifos, Scatchard analysis showed that specific binding sites formed in the imprinted microspheres, the dissociation constants $\left(K_{\mathrm{D}}\right)$ and apparent maximum number $\left(Q_{\max }\right)$ of the MIP were $K_{\mathrm{D}}=7.87 \times 10^{-3} \mathrm{~mol} / \mathrm{L}, Q_{\max }=53.91 \mu \mathrm{mol} / \mathrm{g}$, respectively. The microspheres exhibited highly selective affinity for chlorpyrifos in acetonitrile. The imprinted polymer microspheres could be used as a good material for analytical purposes, such as for enrichment, purification, and analysis of trace organophosphorous pesticides in complex matrix.

\section{References}

[1] USEPA, Preliminary Cumulative Risk Assessment of the Organophosphorus Pesticides, USEPA, Washington, DC, (2001).

[2] Reyzer, M. L., Brodbelt, J. S., Analysis of fire ant pesticides in water by solid-phase microextraction and gas chromatography/mass spectrometry or high-performance liquid chromatography/mass spectrometry. Anal. Chim. Acta, 436, 11-20 (2001).

[3] Díaz-Cruz, M. S., Barceló, D., Highly selective sample preparation and gas chromatographic-mass spectrometric analysis of chlorpyrifos, diazinon and their major metabolites in sludge and sludge-fertilized agricultural soils. J. Chromatography A, 1132, 21-27 (2006).

[4] Ballesteros, E., Gallego, M., Valcarcel, M., Automatic gas chromatographic determination of N-methylcarbamates in milk with electron capture detection. Anal. Chem., 65, 1773-1778 (1993).

[5] Li, H., Liu, Y. J., Zhang, Z. H., Liao, H. P., Nie, L. H., Yao, S. Z., Separation and purification of chlorogenic acid by molecularly imprinted polymer monolithic stationary phase. J. Chromatography A, 1098, 66-74 (2005).

[6] Yan, S. L., Fang, Y. J., Gao, Z. X., Quartz crystal microbalance for the determination of daminozide using molecularly imprinted polymers as recognition element. Biosens. Bioelectron., 22, 1087-1091 (2007).

[7] Alexander, C., Andersson, H. S., Andersson, L. I., Ansell, R. J., Kirsch, N., Nicholls, I. A., O’ Mahony, J., Whitcombe., M. J., Molecular imprinting science and technology: a survey of the literature for the years up to and including 2003. J. Mol. Recognit. 19, 106-180 (2006).

[8] Mahony, J. O., Nolan, K., Smyth, M. R., Mizaikoff. B., Molecularly imprinted polymers - potential and challenges in analytical chemistry Anal. Chim. Acta, 534, 31-39 (2005).

[9] Mayes, A. G., Whitcombe, M. J., Synthetic strategies for the generation of molecularly imprinted organic polymers. Adv. Drug Deliv. Rev., 57, 1742-1778 (2005).

[10] Piletska, E., Piletsky, S., Karim, K., Terpetschnig, E., Turner, A., Biotin-specific synthetic receptors prepared using molecular imprinting. Anal. Chim. Acta, 504, 179-183 (2004).

[11] Zhang, L. Y., Cheng, G. X., Fu, C., Synthesis and characteristics of tyrosine imprinted beads via suspension polymerization. React. Funct. Polym., 56, 167-173 (2003).

[12] Pérez-Moral, N., Mayes, A. G., Comparative study of imprinted polymer particles prepared by different polymerization methods. Anal. Chim. Acta, 504, 15-21 (2004).

[13] Yao, W., Fang, Y. J., Li, G. L., Gao, Z. X. Cheng, Y. Y., Adsorption of carbaryl using molecularly imprinted microspheres prepared by precipitation polymerization. Polym. Advan. Tech. 19, 812-816 (2008) 